\title{
Fluorescence in Best's vitelliform dystrophy, lipofuscin, and fundus flavimaculatus
}

\author{
STEVEN ABBOTT MILLER \\ From the Retina Service, Department of Ophthalmology, University of Wisconsin, Madison, Wisconsin
}

SUMMARY Control photographs, with the Baird Atomic B4 and B5 filters in place prior to fluorescein injection, show exposure of the film corresponding to (1) the small yellow vitelliform lesions at the edge of a disrupted disc, (2) the pseudohypopyon in a vitelliform cyst, (3) orange lipofuscin overlying a malignant melanoma, and (4) some of the flecks in a case of fundus flavimaculatus. Because of transmission overlap between the filters, the relative contribution of reflected light and true autofluorescence is difficult to quantitate. Reflectile structures such as the optic nerve or a white scar were essentially unexposed, but minimal fundus detail was seen. Some parallels exist between lipofuscin and the content of a disrupted vitelliform lesion.

\begin{abstract}
'Autofluorescence', primary, or natural fluorescence, is a physical property in which a light-absorbing substance (fluorophore) re-emits a longer wavelength of light. In fundus fluorescein angiography this optical phenomenon must be differentiated from two types of pseudofluorescence. The first occurs when light reflected from highly reflecting fundus structures excites residual fluorescein in the aqueous and vitreous humour. This excited residual fluorescein in the later stages of angiography may cause a photographic image (Machemer et al., 1970; Archer, 1972). The second type, 'pseudoautofluorescence,' is a reflectile phenomenon in which a faint fundus image, resulting from transmission overlap of the filter pairs, is discernible in control photographs.

Autofluorescence of superficial optic nerve head drusen has been a helpful fluorescein angiographic finding in distinguishing drusen from other causes of disc swelling (Lorentzen, 1966; Sanders and Ffytche, 1967). Many clinicians have noted fluorescence of extensive hard exudate. However, this phenomenon has not been reported in other clinical entities. This report concerns the observation of preinjection fluorescence of (1) the disrupted egg yolk deposits in Best's vitelliform macular dystrophy, (2) orange lipofuscin pigment over a choroidal melanoma, and (3) some of the flecks in a case of fundus flavimaculatus.
\end{abstract}

\section{Patients and methods}

Three members of a family had multifocal, macular

Requests for reprints to: Steven A. Miller, MD, Retina Service, Department of Ophthalmology, University Hospitals, 1300 University Avenue, Madison, Wisconsin 53706, USA and extramacular, Best's vitelliform dystrophy. The diagnosis of Best's dystrophy seemed secure on the grounds of (1) occurrence in a parent and 2 children, (2) characteristic macular lesions, and (3) electrooculogram light-peak/dark-trough ratio between 1.0 and 1.12 in all eyes (normal greater than 1.85).

Fluorescein angiography was performed in the standard manner. Control photographs were taken before fluorescein injection. The filter pair used was the Baird Atomic B4 exciter (less than $2 \%$ of the excited light is transmitted above $5000 \AA$ ) and the B5 barrier (less than $2 \%$ is transmitted below $4950 \AA$ and $0 \cdot 1 \%$ below $4900 \AA$ ). Actual transmission curves of the filters used are graphed in Figs. 1 and 2.

Six other patients with Best's vitelliform dystrophy and fluorescein angiography were reviewed. Because of the colour of the orange yellow egg yolk in Best's dystrophy and the orange lipofuscein pigment seen overlying a choroidal melanoma, colour photographs were reviewed on the 30 most recent cases seen here with the histologically proved diagnosis of malignant melanoma of the choroid. Only 1 case had prominent orange pigment. Fluorescein angiography was available in 6 of 12 patients with the diagnosis of fundus flavimaculatus.

\section{Results}

All yellow disrupted egg yolk spots demonstrated preinjection fluorescence (Figs. $3 a, b, c$ ). This fluorescence occurred in the pseudohypopyon as well as in the circinate distributed yellowish white deposits of the remaining 'scrambled egg' lesions. There was relative fading of the optical phenomenon as the arteriole phase began. The yellow deposit of 
B4 Exciter filter transmission curve

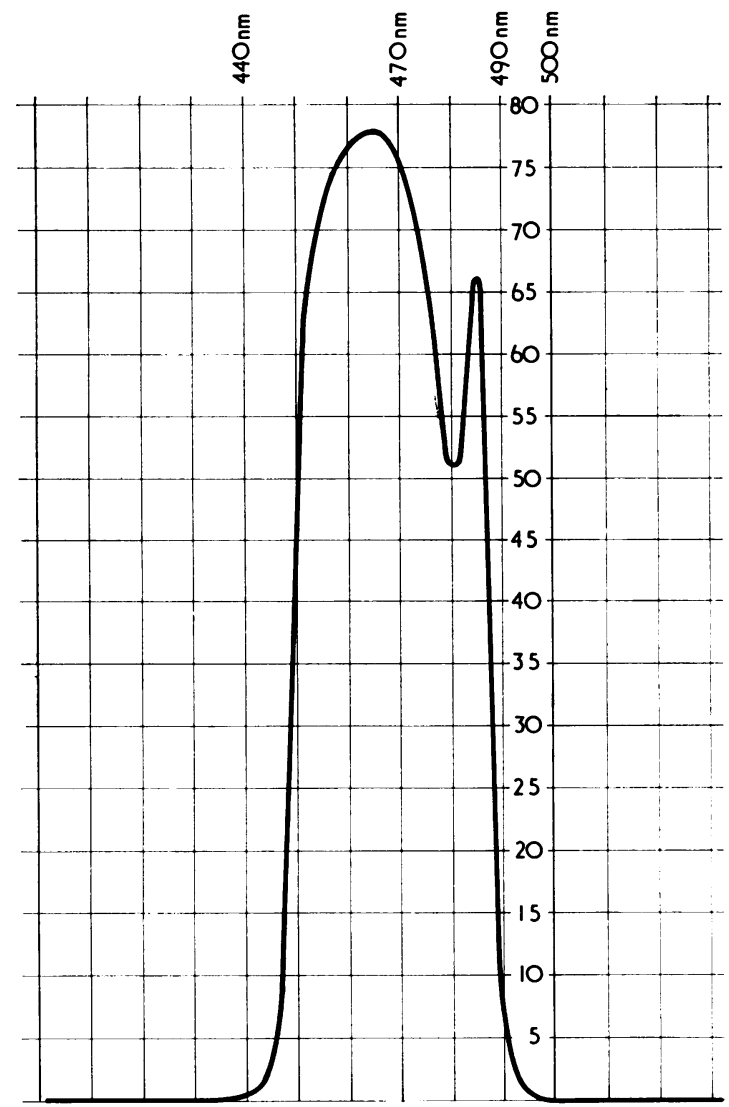

Fig. 1

the pseudohypopyon and the extramacular lesion blocked fluorescence during the fluorescein transit. The circinate yellow deposits acted unpredictably, with some blocking and some transmitting background fluorescence, but most stained. Intact egg yolks, diffuse chorioretinal atrophic lesions, or endstage fibrous scars did not fluoresce. The orange pigment overlying the choroidal melanoma fluoresced in control pictures (Figs. $4 a, b$ ). The pigment blocked fluorescence in the later stages of angiography. One of the 6 patients with fundus flavimaculatus demonstrated preinjection fluorescence (Figs. $5 a, b ; 6 a, b)$. During the dye transit, these flecks showed a retinal pigment epithelial transmission defect, late photographs demonstrated staining of these flecks.

\section{Discussion}

The cases presented demonstrate fluorescence of (1) the small multiple vitelliform deposits at the margin of the larger scrambled atrophic lesion, (2) the pseudohypopyon of a vitelliform cyst, (3) the orange lipofuscin overlying malignant melanoma of the choroid, and (4) some of the flecks in fundus flavimaculatus.

Autofluorescence is a re-emission phenomenona shorter wavelength of light is absorbed and a longer one emitted. It is not a reflectile phenomenon like pseudoautofluorescence-a faint fundus image discernible in control photographs resulting from transmission overlap of the filter pairs. Likewise, pseudofluorescence is excitation of fluorescein within the ocular media from light reflected from reflectile fundus structures such as a white scar.

Indeed, autofluorescence is a common biological phenomenon. Nearly all proteins excited in the 2500 $2800 \AA$ region will fluoresce because of the presence of tryptophan, tyrosine, and phenylalanine. Substances that normally have strong autofluorescence include (Pearse, 1962) collagen, elastic fibres, protein bound $\mathrm{NADH}_{2}$, vitamin $\mathrm{A}$, lipofuscin, and porphyrin. Not unexpectedly, the cornea, nuclear sclerotic lens and intraocular fluids (Krill, 1972) have autofluorescence. However, with conventional angiography this is either minimal or non-detectable.

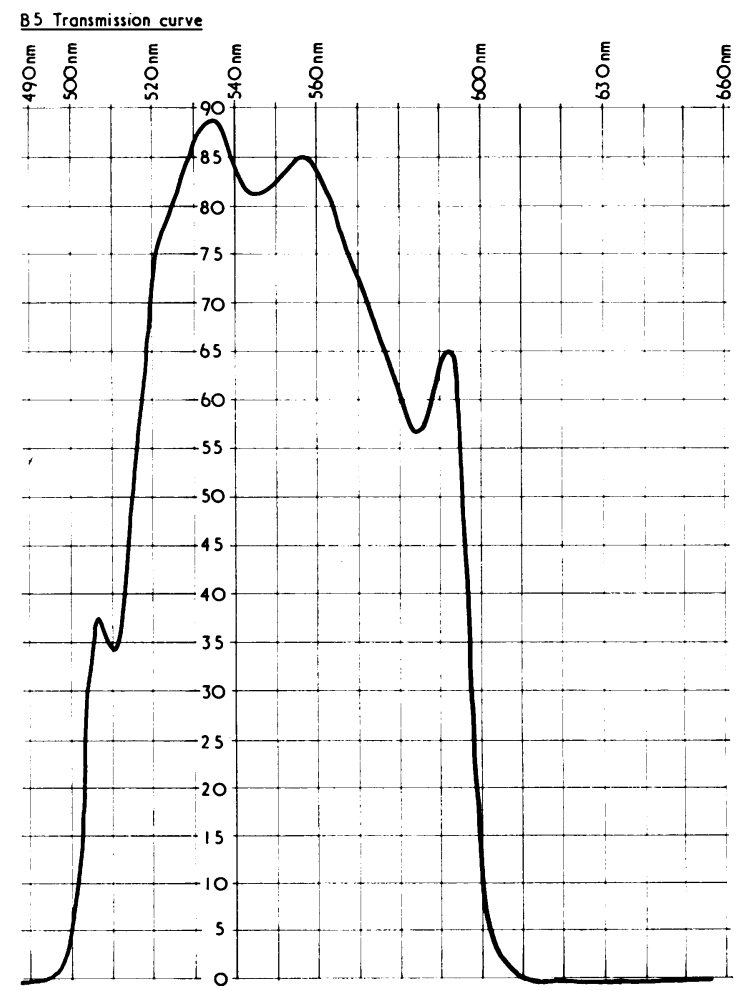

Fig. 2 
Despite the efficient separation between the transmission characteristic of the exciter B4 and the barrier B5 filters, there is nonetheless some lowintensity overlap in transmission, especially of longer wavelengths. Therefore the possibility remains that

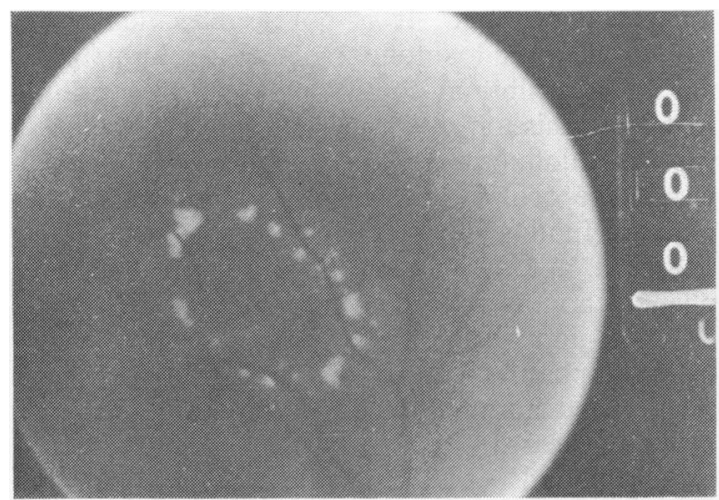

$3 a$

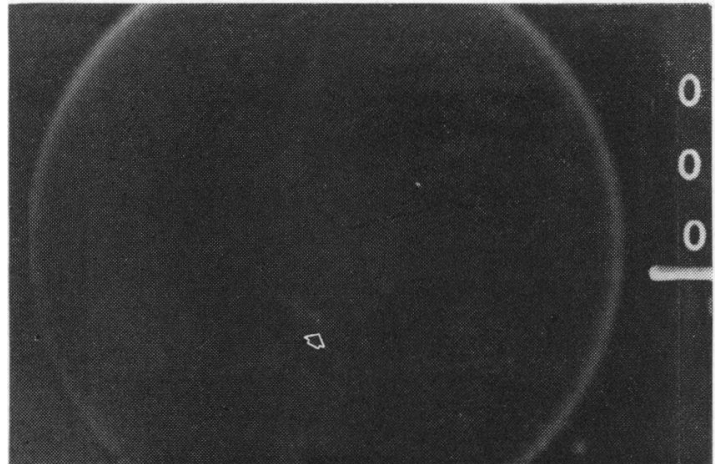

$3 c$

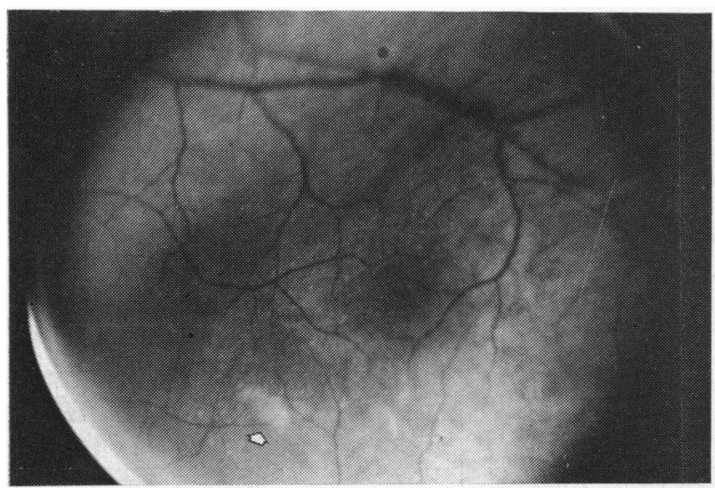

Fig. $4 a$ Choroidal malignant melanoma temporal to the macula in the left eye. The tumour surface is peppered with orange pigment. A confluent patch of orange pigment is present infranasally (arrow) the fluorescing substance in Best's vitelliform dystrophy, lipofuscin granules and some of the flecks in fundus flavimaculatus, is not autofluorescent but rather reflecting enough light to expose the film. Indeed, minimal fundus detail can be seen in most

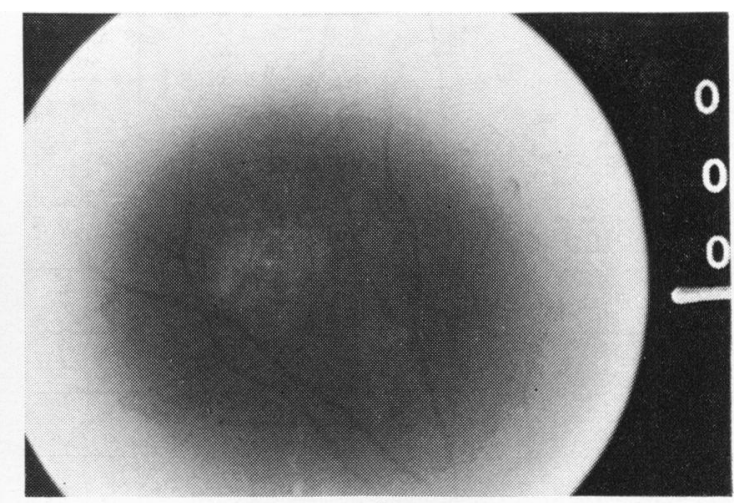

$3 b$

Figs. $3 a, b, c$ Preinjection fluorescence of a variety of macular and extramacular lesions in Best's vitelliform dystrophy

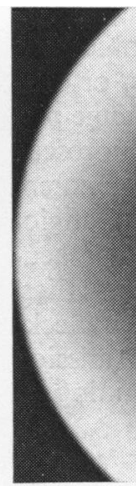

Fig. $4 b$ Preinjection photographs with the Baird Atomic 4 and 5 filters in place demonstrate fluorescence of the orange pigment (arrow) 
of the mounted pictures. This visibility attests to the fact that the filter combination is not ideal. However, those structures that would be expected to reflect light such as the white scar and the optic disc were not visible on the control photographs at the same exposure in which the fluorescing substance was vividly visible. A more perfect filter pair would help resolve the relative contribution of reflected light and autofluorescence. Indeed quantitative absorption and emission spectra of these substances might identify them.

Whether there is any common substance between Best's vitelliform macular dystrophy, fundus flavimaculatus, and lipofuscin is pure speculation. Nonetheless, there are a few interesting common points between Best's dystrophy and lipofuscin as summarised in Table 1.

1. Lipofuscin is orange. $a$. The vitelliform disc is an orange to yellow colour.

2. Lipofuscin granules are most prominent in the

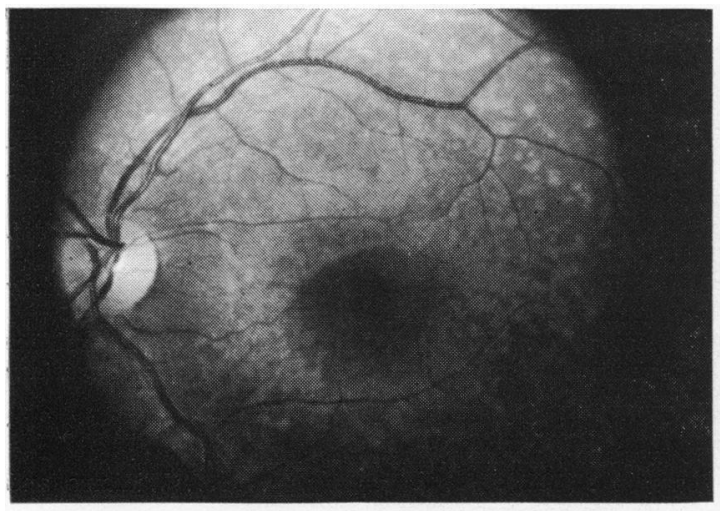

$5 a$

Figs. $5 a, 6 a$ Posterior pole of the left eye and right eye of patient with fundus flavimaculatus

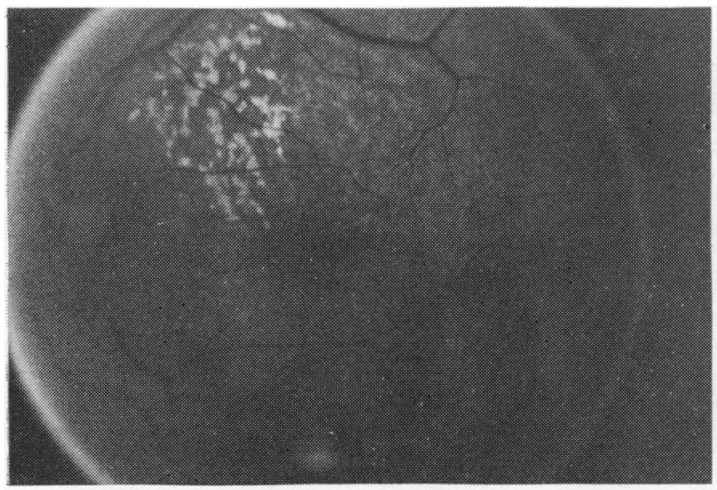

$5 \mathrm{~b}$

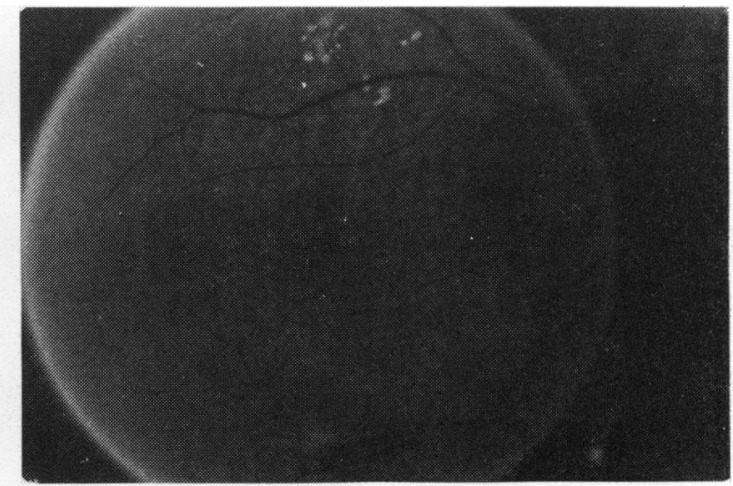

$6 b$ macular region. $a$. The vitelliform disc is almost always macula centred.

3. Lipofuscin increases with age at the posterior pole (Streeten, 1961; Friedman and Tso, 1968). $a$. Vitelliform lesions often become more visible or symptomatic with age (Deutman, 1971) (during its early evolution; after disruption they become less evident).

4. Lipofuscin autofluoresces, with its maximum at 4300 to $4500 \AA$. $a$. The disrupted vitelliform deposit and the lipofuscin overlying a malignant melanoma fluoresce under the same photographic setting.

5. Lipofuscin blocks fluorescein transmission. $a$. The intact vitelliform disc and the pseudohypopyon of a vitelliform cyst block fluorescence.

6. Lipofuscin occurs predominantly within retinal pigment epithelial cells. $a$. Best's dystrophy is thought to be basically a disturbance of the retinal pigment epithelial cell.

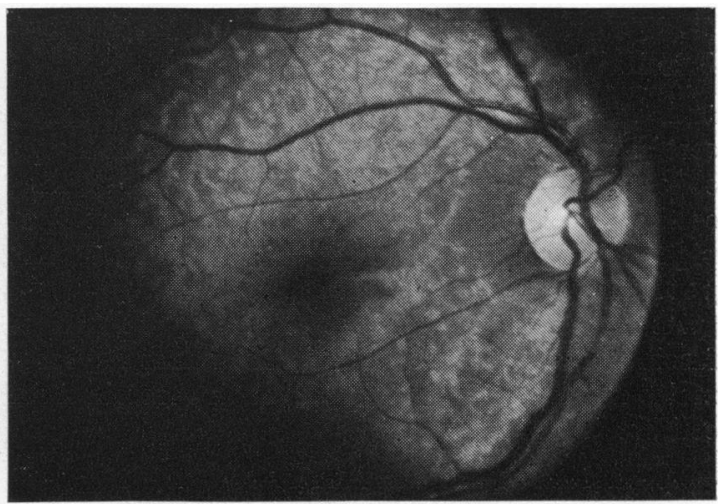

$6 a$

Figs. $5 b, 6 b$ Control photographs show fluorescence of some of the flecks 
Table 1

\begin{tabular}{|c|c|c|}
\hline & Best's vitelliform disc & Lipofuscin \\
\hline Colour & Orange-yellow & Orange \\
\hline Location & Macula centred & Greatest concentration in macula \\
\hline Change with age & $\begin{array}{l}\text { More visible, symptomatic in early stages } \\
\text { (fading late) }\end{array}$ & Increasing density \\
\hline Preinjection fluorescence & $4000-4500 \AA$ & $4300-4500 \AA$ \\
\hline Fluorescein characteristic & Blocks transmission & Blocks transmission \\
\hline Cell involvement & Probably retinal pigment epithelium & Retinal pigment epithelium and macrophages \\
\hline Pathological occurrence & & $\begin{array}{l}\text { 1. Vitamin-E-deficient animals (Mason and Hartsough, } \\
1951 \text {; Hayes, 1974) } \\
\text { 2. Under and within degenerative retina overlying } \\
\text { malignant tumours } \\
\text { 3. Neuronal ceroid-lipofuscinosis }\end{array}$ \\
\hline
\end{tabular}

7. a. Acid-fast yellowish-brown autofluorescent pigments (histochemical characteristics of lipofuscin) were observed to develop in vitamin-E-deficient rats, mink, pigs (Mason and Hartsough, 1951), and monkeys (Hayes, 1974). b. Lipofuscin over a choroidal melanoma is located within proliferating retinal pigment epithelial cells and in macrophages under and within degenerated retina. c. In neuronal ceroid-lipofuscinosis there is early loss of rods and cones, with degeneration of melanin-containing epithelial cells. Characteristic autofluorescent lipopigment granules are found in ganglion, Müller, and photoreceptor cells (Goebel et al., 1977).

At the same time one must bear in mind that lipofuscin is not a single substance but rather a lysosomal oxidative product of lipid or lipoprotein from cell breakdown.

This investigation was supported in part by an NIH Grant EY 00039 from the National Eye Institute.

\section{References}

Archer, D. B. (1972). Fluorescein angiography, in Krill, A. D. (ed.), Hereditary Retinal and Choroidal Diseases, pp. 90-93. Harper and Row: Hagerstown, Maryland.
Deutman, A. F. (1971). The Hereditary Dystrophies of the Posterior Pole of the Eye. C. C. Thomas: Assen, The Netherlands.

Goebel, H. H., Zeman, W., and Damash, E. (1977). An ultrastructural study of the retina in the Jansky-Bielschowsky type of neuronal ceroid-lipofuscinosis. American Journal of Ophthalmology, 83, 70-79.

Friedman, E., Tso, M.O.M. (1968). The retinal pigment epithelium: II Histologic changes associated with age. Archives of Ophthalmology, 79, 315-320.

Hayes, K. C. (1974). Retinal degeneration in monkeys induced by deficiencies of Vit. E or A, Investigative Ophthalmology, 13, 499.

Krill, A. E. (1972). Hereditary Retinal and Choroidal Diseases. Harper and Row: Hagerstown, Maryland.

Lorentzen, S. E. (1966). Drusen of the optic disc. Acta Ophthalmologica, 90, 1-180.

Machemer, R., et al. (1970). Pseudofluorescence. A problem in interpretation of fluorescein angiograms. American Journal of Ophthalmology, 70, 1-13.

Mason, K. E., and Hartsough, G. R. (1951). Journal of the American Veterinary Association, 69, 72.

Pearse, A. (1962). Histochemistry, p. 1117. Williams and Wilkins: Baltimore.

Sanders, M. D., and Ffytche, T. J. (1967). Fluorescein angiography in the diagnoses of drusen of the disc. Transactions of the Ophthalmological Societies of the United Kingdom, 87, 457-468.

Streeten, B. W. (1961). The sudanophilic granules of the human retinal pigment epithelium. Archives of Ophthalmology, 66, 391-398. 